Tropical Journal of Pharmaceutical Research November 2021; 20 (11): 2355-2362

ISSN: $1596-5996$ (print); 1596-9827 (electronic)

(C) Pharmacotherapy Group, Faculty of Pharmacy, University of Benin, Benin City, 300001 Nigeria

Available online at http://www.tjpr.org

Original Research Article

http://dx.doi.org/10.4314/tjpr.v20i11.18

\title{
Prediction of the anti-inflammatory effects of bioactive components of a Hippocampus species-based TCM formulation on chronic kidney disease using network pharmacology
}

\author{
Lingyu Zhang, Sitong Lu, Zhang Hu*, Mingneng Liao, Chengpeng Li, Songzhi \\ Kong \\ Faculty of Chemistry and Environmental Science, Guangdong Ocean University, Zhanjiang 524088, China
}

*For correspondence: Email: huzhangqyx@126.com; Tel: +86-759-238-3300

Sent for review: 19 April 2021

Revised accepted: 18 October 2021

\begin{abstract}
Purpose: To systematically study and predict the therapeutic targets and signaling pathways of Hippocampus (HPC) against chronic kidney disease (CKD) using network pharmacology.

Methods: By combining database mining, literature searching, screening of disease targets, and network construction, the effects of various components of HPC on several proteins related to CKD were predicted and the active compounds were screened. Genes related to the selected compounds were linked using the SEA database. The correlation between CKD and genes was determined using OMIM, DisGenNet, and GeneCards databases. Pathway-enrichment analyses of overlapping genes were undertaken using online databases.

Results: A total of 144 compounds in HPC were identified. Analyses of clusters suggest that the active components of HPC and the target genes against the inflammation caused by CKD were due to 10 compounds and 25 genes. Metascape results showed that these HPC targets are related to CKD inflammation.

Conclusion: The active components of HPC and the target genes against CKD inflammation are involved in multiple signaling pathways, such as AGE-RAGE, TLR, TNF, and NF-KB. This work provides scientific evidence to support the clinical use of HPC against CKD.
\end{abstract}

Keywords: Hippocampus, Chronic kidney disease, Network pharmacology, Paeonol inflammation, AGE-RAGE signaling pathway

\begin{abstract}
This is an Open Access article that uses a funding model which does not charge readers or their institutions for access and distributed under the terms of the Creative Commons Attribution License (http://creativecommons.org/licenses/by/4.0) and the Budapest Open Access Initiative (http://www.budapestopenaccessinitiative.org/read), which permit unrestricted use, distribution, and reproduction in any medium, provided the original work is properly credited.

Tropical Journal of Pharmaceutical Research is indexed by Science Citation Index (SciSearch), Scopus, International Pharmaceutical Abstract, Chemical Abstracts, Embase, Index Copernicus, EBSCO, African Index Medicus, JournalSeek, Journal Citation Reports/Science Edition, Directory of Open Access Journals (DOAJ), African Journal Online, Bioline International, Open-J-Gate and Pharmacy Abstracts
\end{abstract}

\section{INTRODUCTION}

Chronic kidney disease (CKD) is caused by a reduced glomerular filtration rate or kidney damage. Increasingly, CKD has become a severe public health problem worldwide [1,2].
CKD is not only closely related to cardiovascular disease, diabetes and other diseases, but also associated with the increase in mortality and medical care costs. Therefore, early detection and treatment of CKD can reduce economic costs, the burden of advanced kidney disease, 
and the morbidity and mortality of noncommunicable infections.

Hippocampus, also called seahorse, is a collective name for small marine fishes of the Syngnathidae family. Hippocampus species have been used in tonics in traditional Chinese medicine (TCM) formulations. Steroids, metals, amino acids, polypeptides, nucleic acids, minerals, and fatty acids have been isolated from Hippocampus-based TCM formulations [3,4]. According to the theory of TCM, the formula containing Hippocampus materials can nourish the kidney and strengthen the yang. Network pharmacology has been applied to marine-based drugs [5]. Network pharmacology provides a new method to understand the interaction between active components and the related targets of marine-based drugs. However, the mechanisms by which these formulation components affect inflammation and interact or antagonize each other at molecular and network levels are not clear.

In this paper, the active components of HPC and the target genes against CKD inflammation were systematically studied by using network pharmacology. The anti-inflammatory effect of HPC on CKD can be used to provide bioinformatics data for subsequent clinical and basic research on CKD.

\section{METHODS}

\section{Building a database of chemical components}

The studies in the literature were searched by using the keywords of "seahorse" and "Hippocampus" [6]. The databases used for these searches were CNKI (www.cnki.net/), Wanfang (www.wanfangdata.com.cn/index.html), Google Scholar (https://scholar.google.com) and Chemistry Database (www.organchem.csdb.cn).

Using these two keywords, 148 components were documented from the literature of the previous 20 years. The molecular formula of each compound was obtained with PubChem (https://pubchem.ncbi.nlm.nih.gov) or Chemspider (www.chemspider.com) Then, ChemDraw software was employed to draw the structure. SwissADME (www.swissadme.ch) was used to predict ADME parameters. The molecule editor within PubChem, a simplified molecularinput system (SMILES), was employed to screen for the most important components of HPC.

\section{Screening of active compounds}

Considering the cost, length, and complexity of drug development, it is crucial to recognize the bioavailability and potential of drugs. The oral bioavailability (OB), "druglikeness", gastrointestinal tract (GIT) absorption, and ability to cross the blood brain-barrier (BBB) are essential parameters for drug discovery $[7,8]$.

Oral bioavailability is the fractional extent of a drug dose that finally reaches the therapeutic site of action [9]. Druglikeness denotes how "druglike" a substance is concerning properties such as solubility, stability, bioavailability. In general, compounds (and their analogs) screened according to bioavailability are more likely to be used as candidate compounds. Due to the existence of the blood-brain barrier, there are still huge challenges to drugs used in the treatment of brain diseases [10]. Bioavailability is a complex physiologic process affected by several factors.

In the present study, the main active components of HPC were screened based on druglikeness, GIT absorption, and the ability to cross the BBB. Firstly, the chemical structure of each compound was drawn using Chemdraw and introduced into SwissADME, and the typical values of SMILES were obtained. The screening criterion for GIT absorption was defined as "High". The ability to cross the BBB was defined as "Yes". Druglikeness (using the Lipinski Rule of Five) was defined as "Yes, 0 violation". The screening results were based on the potentially active components of HPC.

\section{Selective compounds of HPC and target genes}

Drugs can affect organisms through targeted interactions. Currently, network pharmacology is an efficient approach to decipher the mechanism of drug action [11]. In the process of drug development, the first step is to accurately identify and verify drug-target interactions. Simultaneously, this interaction can help to explain the underlying biological mechanism of action.

In the SwissADME database, according to the selected useful compounds based on SMILES, the similarity ensemble approach (SEA; http://sea.bkslab.org) was used to ascertain the approximate targets of the active components of HPC.

Four publicly available databases: GeneCards v5 (https://www.genecards.org), DisGeNet 7 (www.disgenet.org) [12], DrugBank 5.1.7 (www.drugbank.ca) and OMIM (https://omim.org) were searched, and the target genes associated with CKD, only Homo sapiens proteins linked to 
CKD and the kidney disease online website were selected. Venn diagrams were used to identify and visualize the overlapping genes between compounds and CKD target genes using a free data-analysis platform (www.bioinformatics. com.cn).

\section{Network construction and analyses}

Drug-target networks and disease-target networks can be used to identify the active components and the potential targets, elucidate the mechanism of action, and ascertain the efficacy [13]. After screening the active components of HPC using SwissADME and obtaining the SEA target, the "connection" between the drug and the target was identified. Information on the targets of HPC and CKDrelated diseases was obtained by eliminating the repeated targets. The targets were transformed into a "Gene Symbol" format. The target results were inputted into a database for searching interacting genes/proteins (https://string-db.org). The interaction between the compounds and the overlapping genes was obtained. The interaction network was visualized and analyzed through Cytoscape $\quad 3.7 .0 \quad$ (https://cytoscape.org). Cytoscape is an open-source bioinformatics platform in which "nodes" represent compounds and genes or proteins, and "edges" illustrate interactions between compounds and genes [14]. By setting the "degree value" of compounds or genes, the key active components and critical genes of HPC against CKD were screened out and identified using the topology of the network. The degree value of a compound or target indicates the number of connection points of the compound or gene in the network.

To study the network further, the molecular complexity detection (MCODE) plugin was introduced to generate clusters. MCODE can detect regions of dense connections which represent the molecular complexes in large protein-protein interaction (PPI) networks [15]. MCODE could identify the main centers of HPC and CKD by setting a "k-core". However, the node with the highest score could be found, which was called a "seed" (denoted as a square). The seed could be a key target for this cluster.

Analyses of pathway enrichment of overlapping genes using the Gene Ontology (GO) and Kyoto Encyclopedia of Genes and Genomes (KEGG) database

The related pathways of HPC against CKD were analyzed using $R$ and RGui 4.0.0 (R Project for Statistical Computing, Vienna, Austria; https://www.r-project.org) with a graphical user interface. The critical target for import was prepared in the early stage. Key targets were imported into Metascape (https://metascape.org) [6]. There were 639 entries found in total. In each category, including biological process (BP), cell component (CC), molecular function (MF), the top-20 entries were found $(p<0.05)$. The latest data on pathway-enrichment analysis were from the KEGG database. The cutoff $p$-value was 0.05 . Sixty-five pathways were selected, and the first 10 were chosen.

\section{RESULTS}

\section{Potential active components of HPC}

Four SwissADME models were selected to screen the active components of HPC: bioavailability, druglikeness, crossing the BBB, and GIT absorption. All selected components followed Lipinsky's Rule of Five. A literature search identified 148 compounds in HPC. The SwissADME screening results of these 148 compounds showed that 59 compounds had good activities (Figure 1).

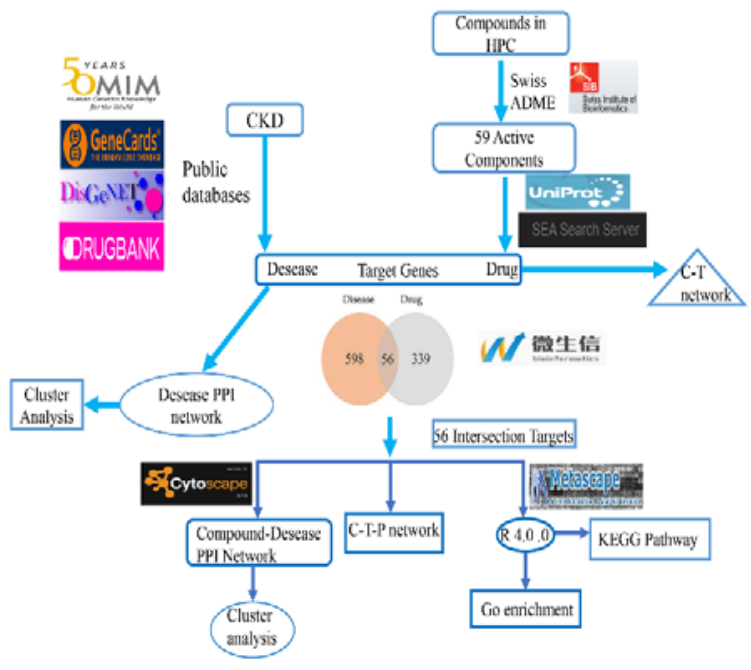

Figure 1. Network pharmacology flow chart

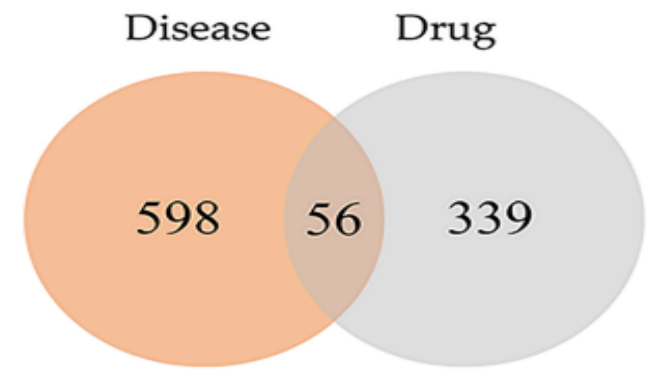

Figure 2: Overlapping genes between 654 Chronic kidney disease (CKD) inflammation-related genes and 56 active compounds-related genes 


\section{Target genes associated with 59 compounds} or CKD

After deletion of duplicated values, 395 target genes were obtained from the 59 compounds in SEA. Interestingly, the 395 target genes were related to only 34 compounds. By searching the databases of Online Mendelian Inheritance in Man (OMIM), GeneCards, DisGeNet, and Drugbank, 654 target genes with a high correlation with CKD were screened. The Venn diagram showed that these 654 CKD-related genes matched the target of the 395 compounds, and 56 overlapping genes could be identified (Figure 2). These 395 compound targets were the targets of 34 out of 59 compounds.

\section{Network construction of drug-component targets}

According to the pharmacokinetic evaluation of HPC and screening using the SEA database, the species were "Homo sapiens", and the related targets of 59 compounds were found. There were 16 HS, 464 QT, 75 TS, 2525 ZFS, and 117 ZT. After deleting duplicate values, 393 targets remained (two targets were independent and were deleted). Among these 393 targets, ZFS had the highest proportion (131 targets). The target with the lowest proportion was HS (Supplementary Information).

To find out how HPC works against CKD, the compound-target (C-T) network was constructed by using 393 targets and 59 components (Figure 3 ). These active components were associated with multiple target points. They resulted in 3182 associations between 59 active components and 393 target points. On the average, each component had 81 target points, and the average composition of each target was 1.37.

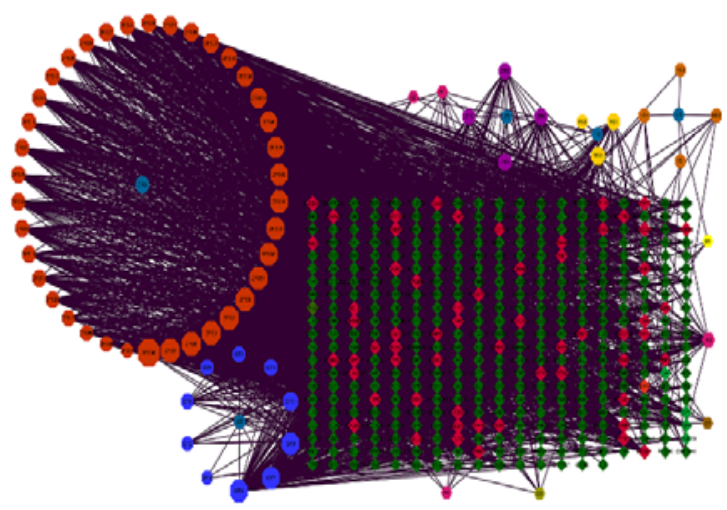

Figure 3: Component-target network of HPC. Different colors and shapes represent other components. The larger the node, the higher the degree of freedom value of the predicted targets by SEA Search Server

\section{Construction of a PPI network}

Based on four gene databases (DisGeNet, OMIM, GeneCards, and DrugBank), there were 654 candidate target points related to CKD. These verified gene IDs were inputted into the STRING database, the species chosen were Homo sapiens, and the confidence score was set at 0.9. The Tab Separated Values (TSV) file was exported, the network was built in the TSV file, and imported into Cytoscape 3.7.0. There were 4886 sides and 654 nodes in the PPI network for CKD (Figure 4). The closer the node was, the more purple it was; the larger the node, the higher the degree of freedom. These findings suggested that these genes were closely related to other genes in the PPI network, and may have an important role in CKD. The 15 proteins with the highest degree of freedom were PIK3CA, PIK3R1, STAT3, APP, MAPK1, IL6, SRC, AKT1, C3, PTPN11, HRAS, JAK2, FN1, JUN, RELA. Their values of degree of freedom were 111,92 , $87,75,73,71,71,71,69,68,66,64$, and 63 respectively.

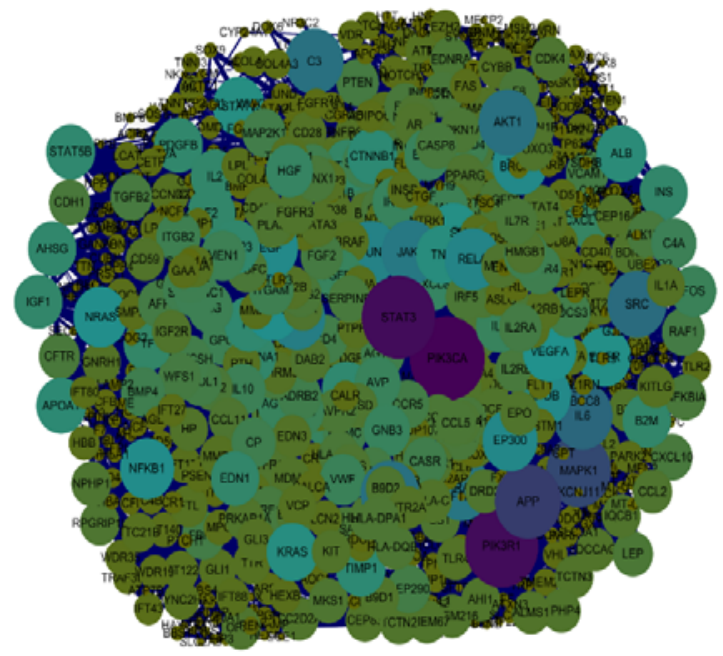

Figure 4: Protein-protein interaction (PPI) network of Chronic kidney disease (CKD)

\section{PPI network of HPC targets and CKD targets}

56 overlapping genes in HPC-CKD targets were found, and two redundant genes were removed. The PPI network comprised 54 nodes and 332 edges (Figure 5). For these 54 target genes, Cytoscape 3.7.0 and STRING 11.0 were used to create a PPI network for HPC-CKD with a confidence cutoff of 0 . 9. Among them, the top10 gene proteins with the highest values were interleukin (IL)6, vascular endothelial growth factor (VEGF)4, toll-like receptor (TLR)4, JUN, proto-oncogene c-Fos (FOS), transcription factor p65 (RELA), IL2, TLR2, estrogen receptor 
(ESR)1 and peroxisome proliferator-activated receptor gamma (PPARG).

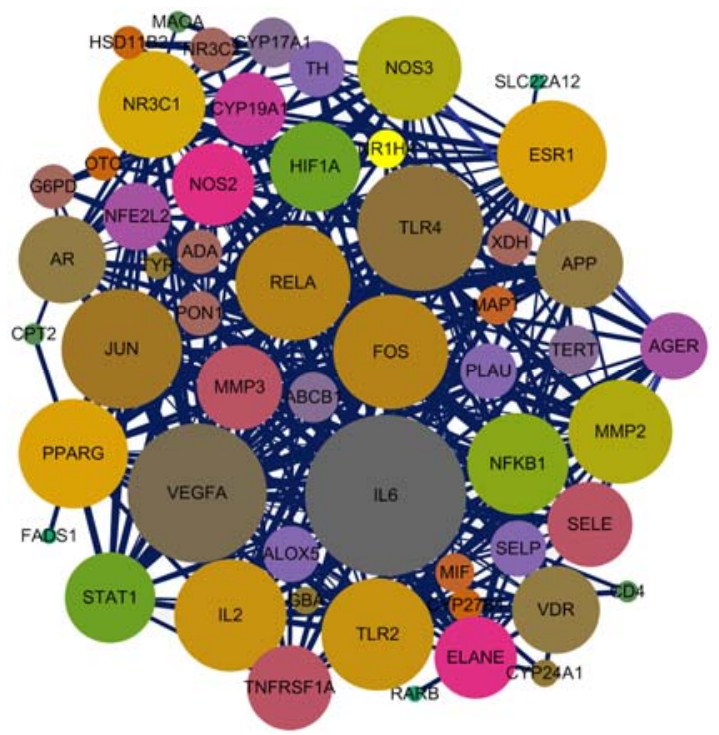

Figure 5: Protein-protein interaction (PPI) network linking 54 target genes related to CKD

\section{Cluster analyses of the PPI network for HPC- CKD}

The PPI network for HPC-CKD (k-core = 2) using the MCODE plugin of Cytoscape revealed two cluster networks (Figure 6, Table 1). In cluster 1 , there were 19 nodes and 122 edges, and the score was 13.556. TLR2/IL1 receptorlike protein was the seed node of cluster 1 , which showed high expression in peripheral-blood leukocytes (especially monocytes), bone marrow, and the spleen. Cluster 2 had six nodes and 11 edges, and its score was 4.4. Toll-like receptors are receptors of the innate immune system responsible for recognizing pathogen-related molecular patterns. Toll-like receptor 2 is the most mixed TLR receptor and can realize the most diverse pathogen-related ways. Toll-like receptor 2 is involved mainly in the inflammatory response and innate immune biological processes.

\section{Analysis of pathway enrichment of overlapping genes using GO and KEGG databases}

Through analyses of pathway enrichment using GO and KEGG databases, the main targets were classified into different functional modules. Metascape was employed to analyze the signaling pathways regulating CKD-related targets, and the results were visualized with $\mathrm{R}$ to determine the biological importance of the main targets. The cutoff value was set to 0.05 . Results showed that the function of multiple targets was closely related to CKD occurrence. Through the GO enrichment analysis of the above network, 639 GO entries were obtained, the top 20 of each category were selected (Figure 7), and the Molecular Function (MF) is shown in Figure 8.

To further verify that certain biological processes associated with target proteins were related to the inflammation observed in CKD, sixty-five pathways ( 0.05 of p-value cutoff) were analyzed using the KEGG database. The results showed that multiple signaling pathways including AGERAGE, TLR, and tumor necrosis factor (TNF) were related to the targets (Figure 8).

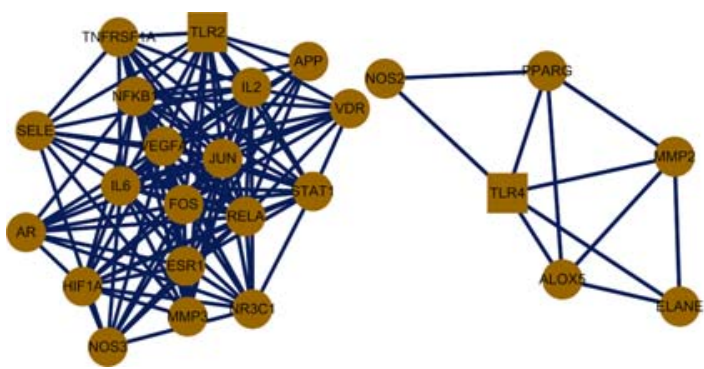

Figure 6: HPC-CKD PPI network. In the HPC-CKD PPI network, the MCODE algorithm study the discovered clusters. The seed nodes of each group are representing as a square

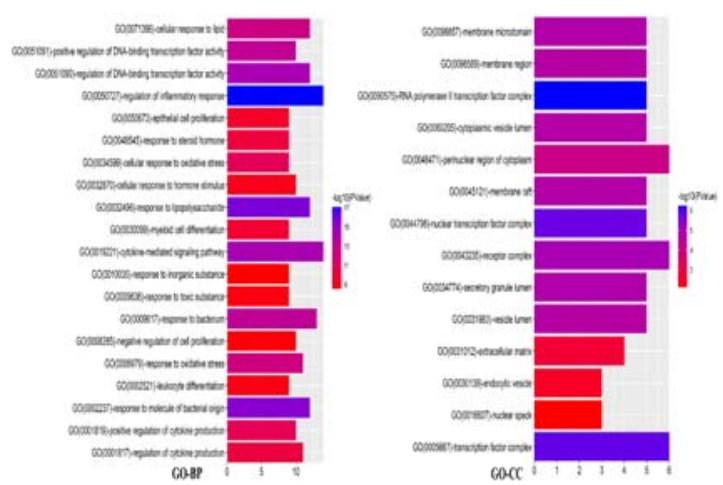

Figure 7: GO Enrichment-BP and CC

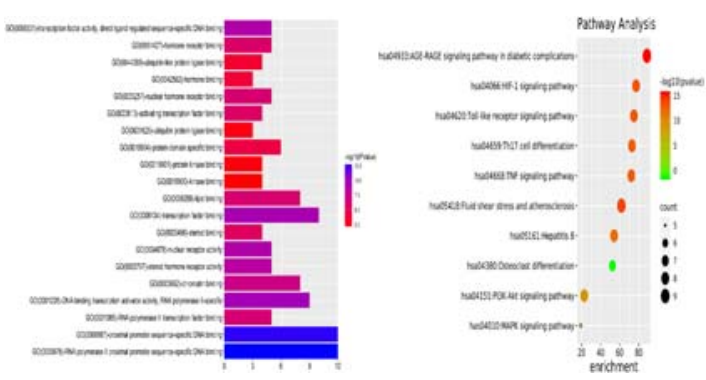

Figure 8: GO Enrichment-MF and Pathway analysis

Trop J Pharm Res, November 2021; 20(11): 2359 
Table 1: Clusters of HPC-CKD PPI network

\begin{tabular}{lcccl}
\hline Cluster & Score & Node & Edge & Gene IDs \\
\hline 1 & 13.556 & 19 & 122 & $\begin{array}{l}\text { APP, IL2, VDR, SELE, STAT1, TNFRSF1A, IL6, } \\
\text { TLR2, ESR1, NR3C1, AR, NFKB1, RELA, NOS3, } \\
\text { HIF1A, VEGF, MMP3, FOS, JUN }\end{array}$ \\
2 & 4.4 & 6 & 11 & NOS2, TLR4, ELANE, PPARG, ALOX5, MMP2 \\
\hline
\end{tabular}

\section{DISCUSSION}

Due to the complexity of human biology, predicting the effects of drugs on human health is challenging. A single target gene rarely regulates a particular disease. Instead, there is multiplelevel interference of interacting networks related to molecular targets, pathways, cells, and tissues [16]. Marine animal-derived medicines are part of traditional Chinese medicine. The active components of marine animal-derived medicines are complex, and it is difficult to clarify the mechanism of action for the treatment of diseases. Network pharmacology provides a new approach to study the action mechanism of multicomponent formulations. Here, the potential bioactive substances related to the important targets of CKD were searched from HPC by using network pharmacology. Through data mining, literature searching, disease target screening, and network construction, the effects of HPC components on several CKD-related proteins were predicted. Figure 9 shows the pathway interaction analysis of the componenttarget network, and the results revealed that HPC was an essential candidate for the treatment of CKD.

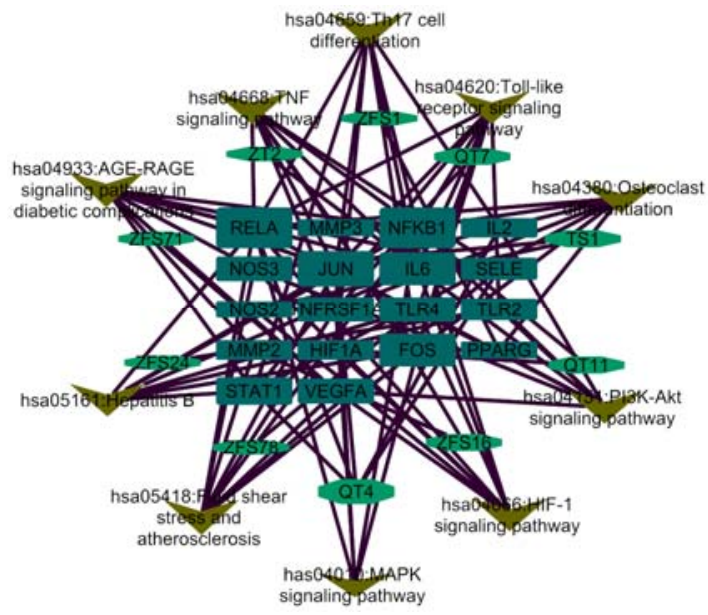

Figure 9: Hippocampus drug composition - kidney disease inflammation target - pathway network

The active components of HPC could act on target proteins through various metabolic pathways. Paeonol from HPC could be employed as an anti-endotoxin in CKD. Two possible mechanisms of action of paeonol from HPC are: (i) inhibit TLR4 expression and phosphorylation of proteins in the nuclear factor-kappa B (NF-KB) signaling pathway, and (ii) restrain the activity of DNA-binding proteins by activating the TLR4-NF$\mathrm{KB}$ signaling pathway [17]. The target genes of paeonol (JUN, E-selectin, RELA, STAT1, and IL2) are also involved in the AGE-RAGE signaling pathway during diabetic complications, and may have therapeutic roles in CKD. Himaya and colleagues found that paeonol isolated from Hippocampus species inhibited activation of BV2 microglia and RAW264.7 macrophages induced by lipopolysaccharide, thereby inhibiting mitogen-activated protein kinase and NF-KB signaling pathways [18]. Nuclear factor-kappa B is a pleiotropic family of transcription factors that regulate inflammation, immunity, as well as the apoptosis, proliferation and differentiation of cells. Nuclear factor-kappa B has been shown to be activated in kidney diseases in experimental animals [19]. Pharmacologic methods can regulate the NF-KB signaling pathway. The active compounds in Hippocampus-based formulations can elicit an anti-inflammatory effect of androstane-4-ene-3,17-dione (ZT2) steroids by inhibiting the transactivation of NF-KB-dependent genes. Therefore, the active components of Hippocampus-based formulations have therapeutic impacts on the inflammation caused by CKD. Cluster analyses demonstrated that TLR2 and TLR4 were the central genes in the main cluster. Toll-like receptors on renal cells contribute to an innate immune response to renal infection, and participate in lupus nephritis [20]. PPI network not only revealed the relationship between HPC and CKD-related targets, but also demonstrated the potential targets from a more objective perspective than that obtained by a clustering technique. Besides, pathwayenrichment analyses showed that all targets interacted with the active components of HPC. Finally, pathway-enrichment analysis showed that the bioactive components of HPC could be expressed through multiple signaling pathways such as AGE-RAGE, TLR, and TNF to aid the regulation of CKD.

\section{CONCLUSION}

The active components of Hippocampus (HPC) and the target genes against the inflammation 
caused by chronic kidney disease (CKD) have been successfully investigated using network pharmacology. The findings show that the active components and the target genes include 10 compounds and 25 genes, respectively. The antiinflammatory effect of HPC on CKD may involve multiple signaling pathways, such as AGERAGE, TLR, TNF, and NF-KB. This work provides scientific evidence to support the clinical use of HPC against CKD.

\section{DECLARATIONS}

\section{Acknowledgement}

The authors gratefully acknowledge the financial support from Guangdong Provincial Natural Science Foundation of China (no. 2016A030308009), Project of Science and Technology Plan of Zhanjiang (nos. 2019A01017 and 2020A01026), Project of Enhancing School with Innovation of Guangdong Ocean University (no. 2017KTSCX090) and Postgraduate Education Innovation Program of Guangdong Ocean University (no. 201926).

\section{Conflict of interest}

No conflict of interest is associated with this work.

\section{Contribution of authors}

We declare that this work was done by the authors named in this article and all liabilities pertaining to claims relating to the content of this article will be borne by the authors. We declare that this work was done by the authors named in this article and all liabilities pertaining to claims relating to the content of this article will be borne by the authors. Lingyu Zhang and Zhang $\mathrm{Hu}$ conceived and designed this work. Lingyu Zhang and Sitong Lu wrote the draft manuscript. Lingyu Zhang collected the data, Sitong Lu and Lingyu Zhang analyzed the data. Mingneng Liao and Chengpeng $\mathrm{Li}$ participated in designing the study. Zhang $\mathrm{Hu}$ and Songzhi Kong wrote and revised the whole manuscript.

\section{Open Access}

This is an Open Access article that uses a funding model which does not charge readers or their institutions for access and distributed under the terms of the Creative Commons Attribution License (http://creativecommons.org/licenses/by/ 4.0) and the Budapest Open Access Initiative (http://www.budapestopenaccessinitiative.org/rea d), which permit unrestricted use, distribution, and reproduction in any medium, provided the original work is properly credited.

\section{REFERENCES}

1. Levey AS, Eckardt KU, Tsukamoto Y, Levin A, Coresh J, Rossert J, Zeeuw DD, Hostetter TH, Lameire $N$, Eknoyan G. Definition and classification of chronic kidney disease: a position statement from Kidney Disease: Improving Global Outcomes (KDIGO). Kidney Int 2005; 67: 2089-2100.

2. Weiner DE, Public health consequences of chronic kidney disease. Clin Pharmacol Ther 2009; 86: 566-569.

3. Wang $Q$, Zhang ZH, Zang XX, Xu GJ. Chemical Studies on Hippocampus histrix Kaup. J China Pharm Univ 1998; 29: 24-25.

4. Chen M, Chen JZ, GeY, Shi SL, Zhang GJ, Chen RB. Research progress on chemical constituents and pharmacological activities of Hippocampus. Chin. Tradit. Herbal Drugs 2017; 48: 4089-4099.

5. Guo Y, Ding Y, Xu F, Liu B, Kou Z, Xiao W, Zhu J. Systems pharmacology-based drug discovery for marine resources: An example using sea cucumber (Holothurians). J Ethnopharmacol 2015; 165: 61-72.

6. Lu, C. Study on Identification and Quality Standard of Hippocampus. Master's thesis, The Second Military Medical University 2015; Shanghai: China.

7. Daina A, Michielin O, Zoete V. SwissADME: a free web tool to evaluate pharmacokinetics, drug-likeness and medicinal chemistry friendliness of small molecules. Sci Rep 2017; 7: 42717.

8. Mahanthesh M, Ranjith D, Raghavendra YJR, Narappa $G$, Ravi M. Swiss ADME prediction of phytochemicals present in Butea monosperma (Lam.) Taub. J Pharma Phytochem 2020; 9: 1799-1809.

9. Chow SC. Bioavailability and bioequivalence in drug development. WIREs Comput Stat 2014; 6: 304-312.

10. Bhowmik A, Khan R, Ghosh MK. Blood brain barrier: a challenge for effectual therapy of brain tumors. BioMed. Res Int 2015; 2015: 1-20.

11. Hopkins AL. Network pharmacology: the next paradigm in drug discovery. Nat Chem Biol 2008; 4: 682-690.

12. Piñero J, Ramírez AJM, Saüch-Pitarch J, Ronzano F, Centeno E, Sanz F, Furlong LI. The DisGeNET knowledge platform for disease genomics: 2019 update. Nucleic Acids Res 2019; 48: 845-855.

13. Gu J, Zhang H, Chen L, Xu S, Yuan G, Xu X. Drug-target network and polypharmacology studies of a Traditional Chinese Medicine for type II diabetes mellitus. Comput Biol Chem 2011; 35: 293-297.

14. Lee AY, Park W, Kang TW, Cha MH, Chun JM. Network pharmacology-based prediction of active compounds and molecular targets in Yijin-Tang acting on hyperlipidaemia and atherosclerosis. J Ethnopharmacol 2018; 221: 151-159.

15. Xu B, Wang Y, Wang Z, Zhou J, Zhou S, Guan J. An effective approach to detecting both small and large

Trop J Pharm Res, November 2021; 20(11): 2361 
complexes from protein-protein interaction networks. BMC Bioinformatics 2017; 18: 419.

16. Berg EL, Yang J, Melrose J, Nguyen D, Privat S, Rosler E, Kunkel EJ, Ekins S. Chemical target and pathway toxicity mechanisms defined in primary human cell systems. J Pharmacol Toxicol Methods 2010; 61: 13-15.

17. Fan HY, Qi D, Yu C, Zhao F, Liu T, Zhang ZK, Yang MY, Zhang LM, Chen DQ, Du Y. Paeonol protects endotoxininduced acute kidney injury: potential mechanism of inhibiting TLR4-NF-KB signal pathway. Oncotarget 2016; 7: 39497.
18. Himaya SWA, Ryu B, Qian ZJ, Kim SK. Paeonol from Hippocampus kuda Bleeler suppressed the neuroinflammatory responses in vitro via NF-KB and MAPK signaling pathways. Toxicol In Vitro 2012; 26: 878-887.

19. Alharbi KS, Fuloria NK, Fuloria S, Rahman SB, Al-Malki WH, Javed Shaikh MA, Thangavelu L, Singh SK, Rama Raju Allam VS, Jha NK, Chellappan DK, Dua K, Gupta G. Nuclear factor-kappa $B$ and its role in inflammatory lung disease. Chem Biol Interact 2021; 345: 109568.

20. Anders HJ, Schlöndorff D. Toll-like receptors: emerging concepts in kidney disease. Curr Opin Nephrol Hypertens 2007; 16: 177-183. 